September 2014, Volume 2

Proceeding of $3^{\text {rd }}$ International Conference on Computation for Science and Technology, ISSN xxxx-yyyy

\title{
Cembranoid Diterpenes as Antitumor: Molecular Docking Study to Several Protein Receptor Targets
}

\author{
Muhammad S. Zubair ${ }^{1,4 *}$, Syariful Anam ${ }^{4}$, Khalid O. Al-Footy ${ }^{1}$, Ahmed Abdel-Lateef ${ }^{2}$, Walied M. Alarif ${ }^{3}$ \\ ${ }^{I}$ Department of Chemistry, Faculty of Science, King Abdul Aziz University, PO.Box 80203, Jeddah 21589, Saudi Arabia. \\ ${ }^{2}$ Department of Natural Products and Alternative Medicine, Faculty of Pharmacy, King Abdul Aziz University, PO.Box 80260, Jeddah \\ 21589, Saudi Arabia. \\ ${ }^{3}$ Department of Marine Chemistry, Faculty of Marine Science, King Abdul Aziz University,PO.Box 80207, Jeddah 21589, Saudi Arabia. \\ ${ }^{4}$ Department of Pharmacy, Tadulako University, Jalan Soekarno Hatta Tondo, Palu 94118, Indonesia.
}

Received: 18 September 2014 / Accepted: 30 November 2014

\begin{abstract}
:
A molecular docking analysis has been carried out on several cytotoxic cembaranoid type diterpenes that have been isolated from soft coral Sarcophyton glaucum i.e sarcophytolol, sarcophytolide B, sarcophytolide C, sarcophine, deoxosarcophine, and cembrene C. All the compounds were investigated using in silico molecular docking with several enzymes and receptor protein targets i.e cyclin-dependent protein kinase 2 (CDK-2), cyclin-dependent protein kinase 6 (CDK-6), protein kinase C (PKC), vascular endothelial growth factor receptor 2 (VEGFR-2), DNA topoisomerase II and tubulin, in order to investigate the potential molecular targets and to correlate the experimental cytotoxicity of these cembranoid compounds. Molecular docking revealed that sarcophytolol $\mathrm{C}$ and deoxosarcophine docked strongly into podophylotoxin binding site of tubulin receptor while the other compounds exhihited selectivity for protein kinase C. The variation of experimental inhibition of concentration $\left(\mathrm{IC}_{50}\right)$ on several cancer cell lines might be due to the difference mechanism of actions of these cembranoid compounds.
\end{abstract}

Key words: Antitumor, cembranoid, docking, PLANTS1.2, sarcophyton glaucum

\section{Introduction}

The advance of knowledge on cancer molecular biology has brought to the new era of anticancer drug discovery. Recently, a new chemopreventive agent must be more selective and less toxic [1]. Therefore, the application of computer on exploration of anticancer molecular mechanism was required to assist many researcher studying the property and selectivity of new anticancer compounds before or after performing experimental testing, both in vitro and in vivo. Considerable attention has been focused on natural compounds capable to inhibit proliferation of cancer cells by inducing apoptosis $[1,2]$.

In this study, cembranoids type diterpenes were in silico studied to investigate the potential molecular target of actions and to correlate with the experimental cytotoxicity. Our previous study have isolated several types of cembranoid compounds from Sarcophyton glaucum i.e sarcophytolol (1), sarcophytolide B (2), sarcophytolide C (3), sarcophine (4), deoxosarcophine (5) and cembrene C (6). Even though, the compounds are likely to induce cellular apoptosis, but their molecular modes of action have not been clearly defined [3]. Therefore, docking molecular analysis have been applied to several enzymes and receptor proteins target responsible for cell cycle, cell proliferation and DNA replication, i.e cyclin-dependent protein kinase 2
(CDK-2), cyclin-dependent protein kinase 6 (CDK-6), protein kinase $\mathrm{C}(\mathrm{PKC})$, vascular endothelial growth factor receptor 2 (VEGFR-2), DNA topoisomerase II and tubulin receptor.

\section{Material and Methods}

Docking was performed with PLANTS1.2 (Konstanz university, Germany) using stochastic optimization algorithms called Ant Colony Optimization (ACO) [4]. Structure Protonation and Recognition System (SPORES) (Konstanz university, Germany), which assigns atom and bond type according to TRIPOS force field convention [5], was used to prepare the protein and ligand structures together with Open Babel version 2.2.3 [6] which employs Monte carlo search with MMFF94 as the force field, for energy minimization of ligand structure. PyMol (www.pymol.org) was used for RMSD calculation and pictures generations [7,8] MarvinSketch (Chemaxon, Netherland) was used for building cembranoid structure [9]. As long as no further explanation, the default settings of each software were used. Simulations were performed on a Linux operating system (Fedora 3.6.114.Fc16 i686) with Intel(R) Core ${ }^{\mathrm{TM}} 2$ Duo CPU T5800@ $2 \mathrm{GHz}$ as processor and 1,93 GB of RAM. 

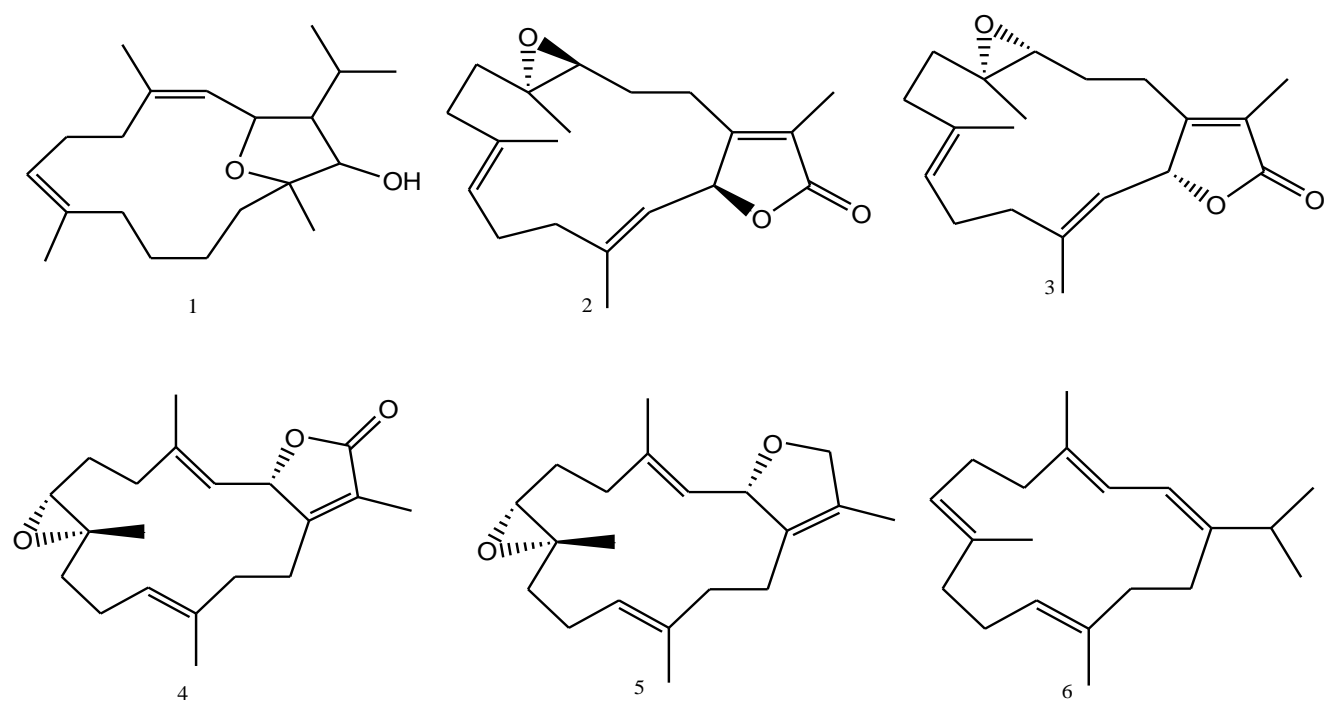

Figure 1. Structure of the studied cembranoid type diterpenes compounds.

Table 1. Target macromolecules, PDB ID, ligand names used in the docking study and the RMSD values

\begin{tabular}{lllc}
\hline Target Macromolecules & PDB ID & Co-crystallized Ligand & RMSD $(\AA)$ \\
\hline Protein CDK2 & 1DI8 & 4-[3-hydroxyanilino]-6,7-dimethoxyquinazoline & 0.54 \\
Protein CDK6 & 1XO2 & Fisetin & 1.56 \\
Protein Kinase C (PKC) & 3TXO & 07u & 1.45 \\
Topoisomerase II enzyme & 1ZXM & Phosphoaminophosphonic acid-adenylate ester (ANP) & 0.49 \\
VEGFR-2 & 2OH4 & GIG & 1.08 \\
Tubulin & 1SA1 & Podophyllotoxin & 0.71 \\
\hline
\end{tabular}

\section{Cembranoids structures preparation}

The structure of cembranoid compounds (figure 1) were built by MarvinSketch (Chemaxon, Netherland). Each structure was then protonated at $\mathrm{pH} 7.4$ and saved as mrv format file. Then, those files were opened again and searched for 10 conformers and saved as mol.2 format file. All the structures subsequently underwent conformational search to find the most stable conformer from 10 seeds, which successively followed by 1000 steps energy minimization using obconformer module in Open Babel. The minimized structure was subjected to SPORES before submitted to the docking simulations using PLANTS1.2.

\section{Receptors and co-crystallized ligands preparation}

All target macromolecule were obtained from the brookhaven Protein Data Bank (www.pdb.org) with the code and co-crystallized ligand presented in table 1 . The PDB files were split into protein and co-crystallized ligands using the splitpdb module on SPORES. All the protein and each co-crystallized ligand were recognized, protonated and stored as mol.2 format file. Preparations of co-crystallized ligands were then continued as the cembranoid structures preparation mentioned above.

\section{Docking Method validation}

To ensure that ligand orientation and positions achieved from the docking simulations were likely to represent valid and reasonable potential binding modes of the inhibitors, the docking methods and parameters used were validated by redocking experiments. All cocrystallized ligands were docked into their protein structure using PLANTS1.2 according to docking protocol constructed and optimized by Istyastono [10]. The binding site in the docking configuration file was defined as $5 \AA$ from the coordinates of the location where the co-crystallized ligands were located in the protein structure. The bind module of PLANTS1.2 was used to automatically identify the binding site. The rmsd value was calculated using rms_cur module in PyMol. This construction procedure was performed iteratively 10 times.

\section{Results and Discussion}

Cembranoids are 14-membered-ring diterpenoid natural products. They play an important role in the biomedical perspective. Antitumor, antimicrobial and antiinflammatory activities have been reported from this type of compounds $[3,11]$. In silico experiment using molecular docking method has been applied to investigate the potential molecular of actions of cembranoids type diterpenes on antitumor/ antiproliferative activity.

In this study, several cembranoid type diterpenes isolated from soft oral Sarcophyton glaucum were docked into each of six different targets. The lowest energy docked of each compounds was selected and taken into account. 
PLANTS1.2 with SPORES and open babel were used together in this docking simulation. Open babel were used to perform stochastic conformational searches followed by energy minimizations to each cocrystallized ligand while the atom and bond types were assigned by SPORES. Followed by docking simulation by PLANTS1.2 using stochastic algorithm, will produce a completely non-deterministic docked pose of each reference ligand (12). The best docking poses of each cocrystallized ligand has RMSD values $<2 \AA$ as presented in Table 1. This meant that constructed protocol docking for each protein/enzyme have prospectively validated.

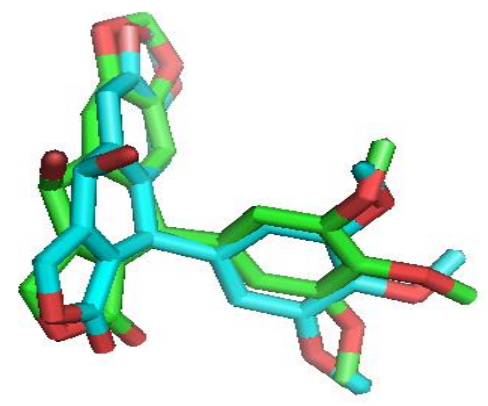

Figure 2. Superimposition of docked pose (green stick figure) and the crystal structure pose (blue stick figure) of the cocrystallized ligand podophyllotoxin in tubulin receptor (1SA1.pdb) with rmsd $0.71 \AA$.

The virtual screening on six different protein/enzyme targets has led to identify tubulin protein receptors and protein kinase $\mathrm{C}$ (PKC) as the potential targets for these cembranoid type diterpenes. The result of docking energy was presented in Table 2. Sarcophytolide C and deoxosarcophine showed docking selectivity for tubulin receptors with docking energies higher than the cocrystallized ligand, $07 \mathrm{u}(-77.4512$ and $75.3408 \mathrm{kcal} / \mathrm{mol}$, respectively). Meanwhile, sarcopytolol, sarcophytolide B, sarcophine and cembrene $\mathrm{C}$ selectively docked with protein kinase $\mathrm{C}$ (figure 3 ) and were the strongest binding ligand for that protein $(-81.6335,-86.6128,-71$. 2449 and $-82.2450 \mathrm{kcal} / \mathrm{mol}$, respectively). Their docking energies were comparable to the co crsytallized ligand podophylotoxin. Saito and co workers [13] reported $\alpha-2,7,11$-cembratriene-4,6-diols $(\alpha$-CBT), the another type of cembranoids, inhibited TPA-stimulated 32Pi incorporation into phospholipids of HeLa cells and a phosporilation of a 47-kilodalton protein that is activated by protein kinase $\mathrm{C}$ (PKC) in human platelets. These results indicated the possible mode of actions of cembranoids compounds might be through inhibition on PKC.

Our research group [3] had reported the cytotoxic activity of these three novel cembranoid compounds against several cancer cell lines. Sarcophytolol exhibited significant cytotoxic effects toward HepG2 (Human hepatoceluller liver carcinoma) with the experimental $\mathrm{IC}_{50}$ values of $20 \pm 0.032 \mathrm{uM}$. Meanwhile, sarcophytolide B showed activity against MCF-7 (Human Breast Adenocarcinoma) with $\mathrm{IC}_{50}$ of $25 \pm$ $0,0164 \mathrm{uM}$. Sarcophytolide C had significant cytotoxic effect to both HepG2 and MCF-7. The variation of these experimental cytotoxic activity of cembranoids might be due to their difference mechanism of actions. Sarcophytolol and sarcophytolide $\mathrm{C}$ had similar cytotoxic activity against HepG2, meanwhile sarcophytolide $\mathrm{B}$ can be considered as less active $\left(\mathrm{IC}_{50}=\right.$ $94.0 \pm 0,022)$. Thus, the less activity of sarcophytolide $B$ might be due to the lower docking energy to tubulin receptor.

The difference in stereochemistry between sarcophytolide B and sarcophytolide C have given difference effect in binding interaction with tubulin receptor in podophylotoxin binding site. Figure 4 showed that cembranoid compounds preferentially docked at the hydrophobic site occupied by $3,4,5$ trimethoxy benzene moiety. The change of configuration from $\mathrm{E}$ form on sarcophytolide $\mathrm{B}$ to $\mathrm{Z}$ form on Sarcophytolide $\mathrm{C}$ led to the difference structural geometry when they bind to tubulin receptor generating the higher interaction with protein. This docking study identified the furan ring with the lactone group can be a pharmacophore group occupying the hydrophobic site of tubulin receptors and creating polar interaction with CYS240.

Table 2. Docking energy of cembranoid type diterpenes compounds docked with target macromolecules

\begin{tabular}{lcccccc}
\hline \multirow{2}{*}{ Cembranoid Compounds } & \multicolumn{5}{c}{ Target Macromolecule, Docking Energy (Kcal/mol) } \\
\cline { 2 - 7 } & 1DI8 & 1XO2 & 3TXO & 1ZXM & 2OH4 & 1SA1 \\
\hline Sarcophytolol & -76.8621 & -71.2608 & $\mathbf{- 8 1 . 6 3 3 5}$ & -80.2850 & -73.8077 & -73.3075 \\
Sarcophytolide B & -68.9742 & -74.3430 & $-\mathbf{8 6 . 6 1 2 8}$ & -67.7163 & -69.6695 & -71.1988 \\
Sarcophytolide C & -73.7127 & -71.0874 & -73.8284 & -79.3110 & -74.7806 & -77.4512 \\
Sarcophine & -61.2233 & -59.4828 & -71.2449 & -62.3230 & -67.5124 & -71.0381 \\
Deoxosarcophine & -61.4020 & -67.5805 & -68.1897 & -67.9147 & -67.7554 & -75.3408 \\
Cembrene C & -79.0655 & -82.1601 & -82.2450 & -66.5433 & -75.1866 & -75.9790 \\
Co-crystallized ligand & -88.3701 & -91.0738 & -93.6941 & -163.7040 & -136.4820 & -74.7457 \\
\hline
\end{tabular}

${ }^{a}$ Ligands showing selective (significantly stronger docking than average for all proteins) docking energies are highlighted in blue bold. 


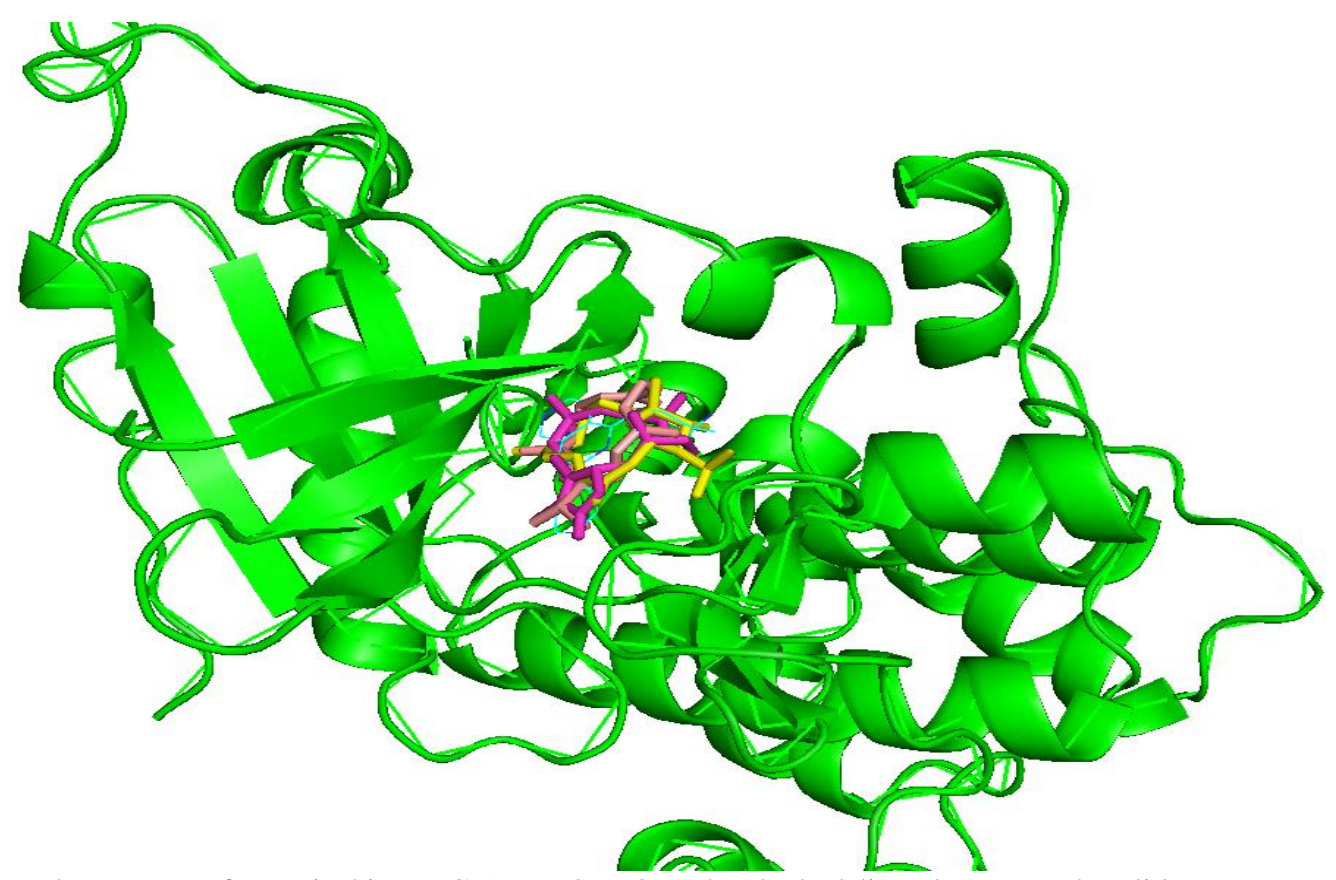

Figure 3. The crystal structure of protein kinase C (PDB 3TXO). The docked ligand are sarcohytolide B (magenta stick figure), sarcophytolol (yellow stick figure) and cembrene $\mathrm{C}$ (brown stick figure). The co-crystallized ligand, 07u, is shown as a line figure.

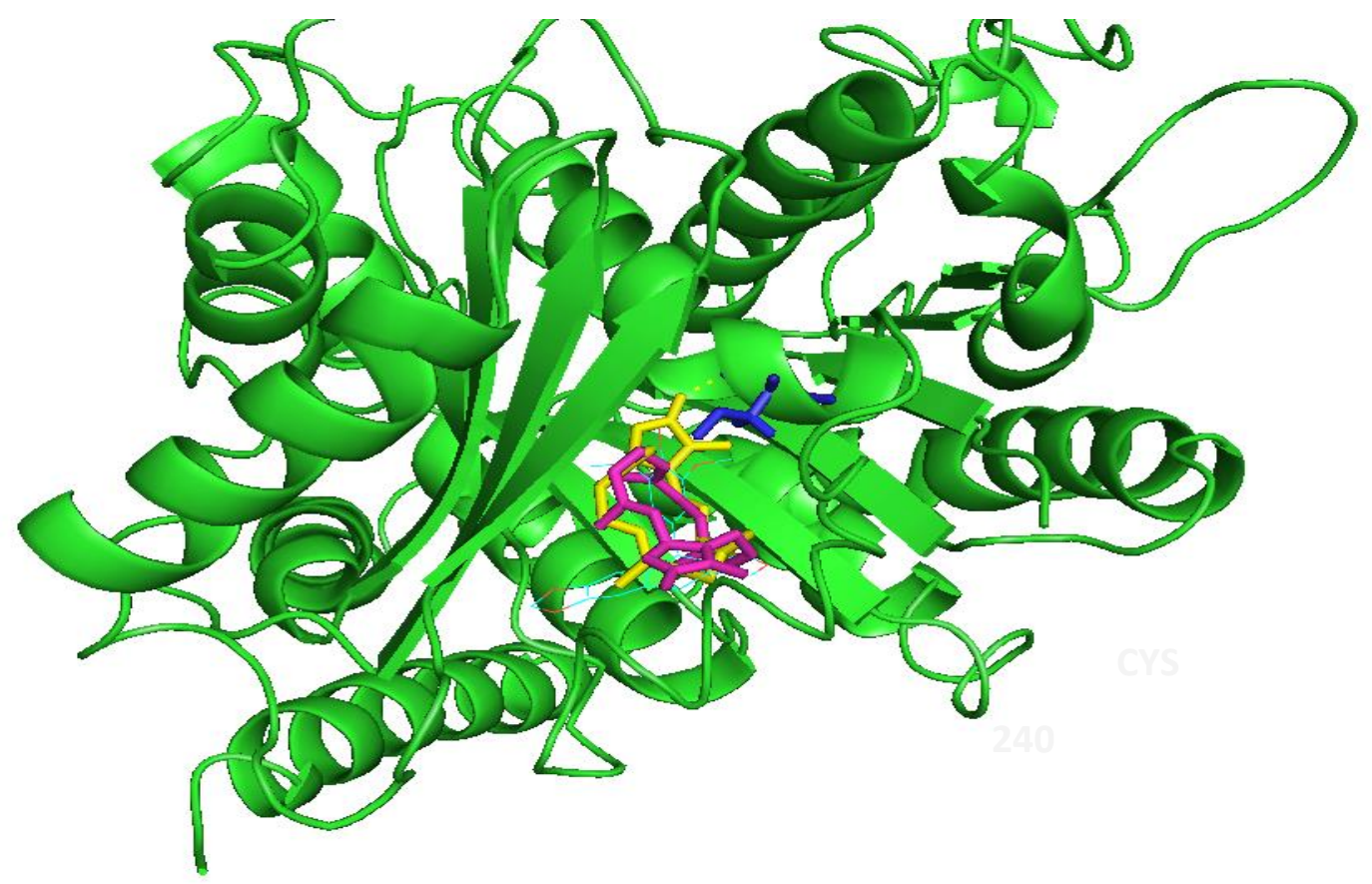

Figure 4. Sarcophytolide B (magenta stick figure) and sarcophytolide C (yellow stick figure) in podophylotoxin (line figure) binding site in tubulin crystal structure (PDB 1SA1).

\section{Conclusions}

This in silico molecular docking study has identified tubulin and protein kinase C (PKC) as potential molecular targets for cembranoid type diterpenes. The variation of their experimental cytotoxic activity in several cancer cell lines might be due to their difference in mechanism of actions, led to further investigations.

\section{References}

[1] N. Phosrithong and J. Ungwitayatorn, Molecular docking study on anticancer activity of plant-derived natural products, Med. Chem. Res., 19, 2010, 817-835.

[2] W.N. Setzer and I.V. Ogungbe, In-silico investigation of antitrypanosomal phytochemicals from Nigerian medicinal plants, PLoS. Negl. Trop. Dis., 6(7), 2012, e1727.

[3] S.S. Al-Lihaibi, W.M. Alarif, A.A Lateef, S.N. Ayyad, A.B. Abdel-Naim, F.E. El-Senduny, and F.A. Badria, Three new cembranoid-type diterpenes from Red Sea soft coral Sarcophyton glaucum: Isolation and antiproliferative 
activity against HepG2 cells, Eur. J. Med. Chem., 81, 2014, 314-322.

[4] O. Korb, T. Stutzle, and T.E. Exner, Empirical scoring functions for advanced protein-ligand docking with PLANTS, J. Chem. Inf. Model., 49(1), 2009, 84-96.

[5] T. ten Brink and T. E. Exner, The influence of protonation states on protein-ligand docking results, J. Chem. Inf. Model., 49(6), 2009, 1535-1546

[6] N.M. O'Boyle, M. Banck, C.A. James, C. Morley, T. Vandermeersch, and G.R. Hutchison, Open Babel : An open chemical toolbox, J. Cheminform., 3, 2011, 33.

[7] W.L. DeLano, 2002, The PyMOL User's Manual, Palo Alto, CA, USA.: DeLano Scientific.

[8] D. Seeliger, and B.L. de Groot, Ligand docking and binding siteanalysis with PyMOL and Auto dock/Vina, J. Comput. Aided Mol. Des., 24(5), 2010, 417-422.

[9] E.R. Bennett, J. Clausen, E. Linkov, and I. Linkov, Predicting physical properties of emerging compounds with limited physical and vhemical data : QSAR model uncertainty and aplicability to military munitions, Chemosphere, 77(10), 2009, 1412-1418.

[10] N. Yuniarti, Z. Ikawati, E.P. Istyastono, The importance of ARG513 as a hydrogen bond anchor to discover COX-2 inhibitors in a virtual screening campaign, Bioinformation, 6(4), 2011, 164-166.

[11] G.H Wang, H.C Huang, J.H. Su, C.Y. Huang, C.H. Hsu, Y.H. Kuo, and J.H. Sheu, Crassocolides N-P, Three cembranoids from the Formosan soft coral Sarcophyton crassocaule, Bioorg. \& Med. Chem. Lett., 21, 2011, 72017204.

[12] E.P. Istyastono, Construction and optimization of structurebased virtual screening protocols to identify cyclooxygenase-1 inhibitors using open babel, SPORES and PLANTS, Indo. J. Chem., 12(2), 2012, 141 - 145.

[13] Y. Saito, H. Nishino, D. Yoshida, S. Mizusaki, and A. Ohnishi, Inhibition of 12-0-tetradecanophorbol- 13-acetatestimulated 32Pi incorporation into phospho lipids and protein phosphorylation by 2,7,1 1-cembratriene-4,6-diol, an antitumorpromoting agent, Oncology, 45(2), 1988, 122-126. 